Über Fettbestimmung im Brot.

Von

\section{Mats Weibull.}

Da ich neulich einige Analysen von Brot sammt den beim Brotbacken benutzten Materialien ausführte, ergab sich immer ein niedriger Gehalt von Fett im Brot (auf Trockensubstanz berechnet) als im Mehle, welches zum Brotbacken benutzt war. Ich verglich daher die in der Literatur zugänglichen Brotanalysen mit den Analysen der entsprechenden Mehlarten; auch hier, wenn man die Analysen auf Trockensubstanz umrechnet, findet man immer weniger Fett im Brot als im Mehle. Beispielsweise fübre ich hier einige Durchschnittszahlen von mehreren Analysen an, die aus König's bekannter Arbeit') genommen sind.

\begin{tabular}{clcc} 
& & \multicolumn{2}{c}{ Fett } \\
Feines & Weizenmehl & \multicolumn{1}{c}{1,08 Proc. } \\
- & Weizenbrot & $0,71-$ \\
Grobes Weizenmehl & $1,56-$ \\
- & Weizenbrot & 0,74 & - \\
Feines & Roggenmehl & 1,91 & - \\
- & Roggenbrot & 0,75 & -
\end{tabular}

Vereinzelt kommen sogar Brotanalysen vor, die einen Fettgehalt von 0,10 Proc. zeigen, während doch im Mehle ein Gehalt von 0,44 Proc. das Minimum ist.

Meine Beobachtung war also nicht vereinzelt, entweder geht also beim Backen eine beträchtliche Menge Fett verloren, was aber unwahrscheinlich ist, da die Temperatur der Brotkrumen beim Backen kaum $100^{\circ}$ überschreitet ${ }^{2}$ ), oder die Bestimmungen des Fettgehalts sind in den meisten Fällen fehlerhaft. Einige Versuche, die ich hier anführen werde, haben diese letztere Ansicht bestätigt.

Wenn man in gewöhnlicher Weise das Brotpulver in Soxhlet's Extractionsapparat behandelt, wird das Fett sehr langsam ausgezogen, ja man bekommt, wenn man gewöhnlichen Äther benutzt, nie constantes Gewicht. $5 \mathrm{~g}$ gepulvertes Brot, dessen Fettgehalt $z u$ 1,47 Proc. berechnet war, lieferte beispielsweise nach der hier angeführten Extractionszeit folgende Extractmenge:

\begin{tabular}{|c|c|c|}
\hline Nach 6 & Stunden & 0,29 Proc. \\
\hline & - & 0,41 \\
\hline & - & 0,98 \\
\hline 80 & - & 1,10 \\
\hline 110 & - & 1,14 \\
\hline
\end{tabular}

Nachher wurde das Brotpulver nochmals äusserst fein gepulvert und mit Äther behandelt. Jetzt wurde ausgezogen:

1) Die menschl. Nahrungs- und Genussmittel. Berlin 1883, Bd. 1, S. 382, 420; Bd. 2, S. 73, 118

2) Birnbaum: Das Brotbacken. Braunschweig 1879, S. 122.

$$
\begin{aligned}
& \text { nach } 24 \text { Stunden } \quad 0,24 \text { Proc. } \\
& \text { - } 50 \text { - } 0,29
\end{aligned}
$$

In zusammen 160 Stunden waren also 1,43 Proc. Fett ausgezogen oder beinahe die berechnete Menge. Der Auszug enthielt neben Fett eine beträchtliche Menge von Traubenzucker, der von dem geringen Wassergehalt des Äthers ausgezogen war. Ich versuchte jetzt die Extraction dadurch zu erleichtern, dass ich das Brotpulver mit Bimsstein sehr fein zerrieb, das Pulver schichtenweise mit Kaolin in die Extractionshülse einfüllte und mit wasserfreiem Äther behandelte. Auch jetzt war nach 32 Stunden constantes Gewicht nicht zu bekommen, und nur $3 / 4$ von dem berechneten Fettgehalt ausgezogen.

Da es also nicht möglich war, durch Pulvern das Brot für Fettextraction geeignet zu machen, so musste beim Backen das Fett von Dextrin und Stärke sehr dicht umschlossen werden, und sich daher der Einwirkung des Äthers entziehen. Wenn man dagegen Stärke und Dextrin vollständig in Lösung bringt, so wird das Fett in der entstandenen Flüssigkeit vertheilt und kann in gleicher Weise bestimmt werden wie in Milch. Diese Voraussetzung hat sich als richtig erwiesen; um eine genaue Bestimmung des Brotfettes zu erreichen, verfährt man in folgender Weise.

Man übergiesst 1 bis $\mathbf{3} \mathrm{g}$ gepulvertes Brot $^{3}$ ) in einem kleinen Becher mit 15 bis 30 cc Wasser, wozu 10 Tropfen verdünnte Schwefelsäure gesetzt sind. Die Flüssigkeit wird nachher wenigstens 1 Stunde gekocht, während mit einem Glasstabe umgerührt wird; man neutralisirt allmählich und vollständig mit Marmorpulver und breitet die dickflüssige Masse wie bei der Milchfettbestimmung auf Papier aus, welches man in einem Trockenschrank an Metalldrähten hängen hat. Mit Hülfe von etwas entfetteter Baumwolle kann man allen Rückstand von dem Becher entfernen; das Papier mit der Brotmasse und Baumwolle ist nach Erhitzen bis $100^{\circ}$ wäbrend 2 bis 3 Stunden vollkommen wasserfrei und kann jetzt in einen gewöhnlichen Extractionsapparat (in welchem sich eine Unterlage von Baumwolle befindet) eingeführt und mit wasserfreiem Äther extrahirt werden. Wenn ein geräumiger Extractionsapparat verwendet wird, kann in dieser Weise alles Fett in 8 Stunden vollständig ausgezogen werden. Benutzt man gewöhnlichen Äther, so geht allmählich etwas

3) Nach der Grösse des für Fettextraction benutzten Apparates; in die gewöhnlichen Soxblet'schen Hebelapparate kann man passend nicht mehr als 1,5 g nehmen. 
Traubenzucker in die Lösung und constantes Gewicht kann nicht erreicht werden.

Zur Prüfung der Methode wurde ein Brot (von Roggenmehl und Milch gebacken) untersucht, dessen Fettgehalt durch Analysen der Bestanảtheile zu 1,47 Proc. berechnet war. Die folgende Tabelle gibt an: a) die eingewogene Brotmenge, I. das Resultat der ersten Extraction, nämlich sowohl den Extractgehalt als die Extractionszeit, II. die zweite, III. die dritte Extraction und b) den Extractgehalt in Proc.

\begin{tabular}{c|c|c|c|c|c|c|c|c|c}
\hline \hline No. & a) & \multicolumn{2}{|c|}{ I. } & \multicolumn{2}{|c|}{ II. } & \multicolumn{2}{|c|}{ III. } & b) \\
& g & St. & g & St. & g & St. & g & Proc. \\
\hline 1 & 1 & 8 & 0,0149 & 2 & 0 & - & & 1,49 \\
2 & 1,5 & 2 & 0,0196 & 6 & 0,0024 & 3 & 0 & 1,47 \\
3 & 2,5 & 6 & 0,0334 & 4 & 0,0024 & 3 & 0 & 1,43
\end{tabular}

Anm. Das Fett war vollkommen löslich in einer kleinen Menge wasserfreien Äthers und enthielt eive etwas geringere Menge freier Säuren als das Mehlfett.

Auch wurde die Methode an dem in Südschweden gewöhnlichen Grofbrod (d. h. Schwarzbrot) geprüft. Die Materialien konnten nicht analysirt werden, da das Brot am Markt in Lund gekauft war. Der Fettgehalt dürfte doch die Mittelzahl für grobes Roggenmehl nur wenig untersteigen; nach $\mathrm{König} \mathrm{ist} \mathrm{diese} \mathrm{Zahl} \mathrm{2,42,} \mathrm{auf} \mathrm{Trockensub-}$ stanz berechnet.

I. Zuerst wurden $3,5 \mathrm{~g}$ wasserfreies Brotpulver direct mit wasserfreiem Äther extrahirt. Dabei wurden in 13 Stunden 1,21 Proc. Extract, in weiteren 14 Stunden nur 0.10 Proc. Extract bekommen, also wïhrend 27 Stunden 1,31 Proc. Jetzt wurde das extrahirte Brotpulver in oben beschriebener Weise mit verdünnter Schwefelsäure behandelt; dabei wurden in 12 Stunden 0,97 Proc. Fett extrahirt, gleichzeitig war auch constantes Gewicht erhalten und im Ganzen war also 2,28 Proc. Fett bekommen.

II. Wenn gepulvertes, wasserfreies Brot sofort nach oben beschriebener Methode behandelt wurde, bekam ich folgendes Resultat:

\begin{tabular}{|c|c|c|c|c|c|c|c|c|c|c|}
\hline \multirow{2}{*}{ No. } & a) & \multicolumn{2}{|r|}{ I. } & \multicolumn{2}{|c|}{ II. } & \multicolumn{2}{|r|}{ III. } & \multicolumn{2}{|c|}{ IV. } & \multirow{2}{*}{$\begin{array}{c}\text { b) } \\
\text { Proce }\end{array}$} \\
\hline & $\mathrm{g} \mid$ & St. & $\mathrm{g}$ & St. & $\mathrm{g}$ & St. & $\mathrm{g}$ & & $\mathrm{g}$ & \\
\hline $\begin{array}{l}1 \\
2\end{array}$ & $\begin{array}{l}3 \\
3\end{array}$ & $\begin{array}{l}7 \\
7\end{array}$ & $\begin{array}{l}0,0619 \\
0,0646\end{array}$ & $\begin{array}{l}4 \\
4\end{array}$ & $\begin{array}{l}0,0052 \\
0,0040\end{array}$ & $\begin{array}{l}3 \\
3\end{array}$ & {$\left[\begin{array}{c}0,0005 \\
0\end{array}\right.$} & & 0 & $\begin{array}{l}2,2 \\
2,2\end{array}$ \\
\hline
\end{tabular}

Derselbe Fettgehalt wurde also wie früher bekommen. Auch hier wurde freie Säure nur in sehr geringen Mengen extrahirt, denn für $1 \mathrm{~g} \mathrm{Fett}$ wurden $13 \mathrm{cc} / 1 / 10$ Normalnatron verbraucht. Dies entspricht der $\mathrm{Zahl}$ des Roggenmehles; die im Schwarzbrot bei der Gährung entstandenen Säuren werden also von dem Kalk gebunden und kommen nicht in die ätherische Lösung.

Aus den obigen Versuchen scheint hervorzugehen, dass eine gewisse Verschiedenheit besteht bezüglich der für Extraction nöthigen Zeit; diese beruht wesentlich auf dem benutzten Extractionsapparat. So ist die verschiedene Zeit bei den letzten Analysen dadurch zu erklären, dass ich im ersten Falle einen kleinen Apparat benutzte, wo das zur Extraction eingeführte Papier, Brot und Baumwolle beinahe den halben Apparat erfüllte, und der Äther daher diese nur sparsam durchdringen konnte, während im letzten Falle ein etwas grösserer Apparat benutzt wurde.

Die oben angefübrte Annahme, dass beim Backen das Fett des Mehles von den übrigen Bestandtheilen des Brotes eingeschlossen wird, hat sich also bestätigt. Beim Kochen mit verdünnter Schwefelsäure werden die Hauptbestandtheile des Brotes, die Stärke und Dextrin gelöst, das Fett also frei gemacht und in grossen Tropfen gesammelt, welches bei dem nachher folgenden Eintrocknen von Papier, Gips und überschiessendem Marmor aufgenommen wird. Übrigens wird das Fett nicht allein in dieser intensiven Weise von Stärke u. dgl. eingebettet, auch die Kleberstoffe des Brotes sind in ähnlicher Weise mit Stärke gemischt. Auch wenn man das Brot mit Malzauszug digerirt, gelingt es nämlich nicht, die Stärke so rollständig zu lösen, dass eine reine Proteïnsubstanz übrig bliebe ${ }^{4}$.

Alnarps chemisches Laboratorium. Juli 1892.

\section{Welches sind die zweckmässigsten Elektricitätsquellen zur Elektrolyse.}

Von

\section{H. Nissenson und C. Rüst.}

Bei der Ausführung der quantitativen Analyse durch Elektrolyse stellt man verschiedene Anforderungen an die Stromquellen. Der Strom soll möglichst constant sein, ebenso soll er seine Spannung während der Dauer des Versuches nicht wesentlich ändern. Je nach dem Metalle, welches auf elektrolytische Weise bestimmt werden soll, ist im einen Falle ein starker Strom nothwendig, im anderen dagegen soll das Metall durch einen ganz schwachen Strom ausgefällt werden. Für die Bestimmung der Metalle durch Elektrolyse sind eine ganze Reihe verschiedener Vorschriften für ein und dasselbe Metall gegeben, je nachdem man es aus saurer, neutraler, alkalischer Lösung,

4) Siehe Birnbaum a. a. O. S. 253. 